\title{
TWISTS OF MATRIX ALGEBRAS AND SOME SUBGROUPS OF BRAUER GROUPS II
}

\author{
WeNCHEN ChI
}

\begin{abstract}
We consider some subgroups of Brauer groups arising from twists of matrix algebras by some continuous characters. Explicit descriptions of these subgroups are given in terms of Gauss sums of Dirichlet characters.
\end{abstract}

\section{INTRODUCTION}

Let $\overline{\mathbb{Q}}$ be a fixed algebraic closure of the field of rational numbers $\mathbb{Q}$, and let $G_{\mathbb{Q}}=$ $\mathrm{Gal}(\overline{\mathbb{Q}} / \mathbb{Q})$ be the Galois group furnished with the Krull topology. For an arbitrary field $F$ in $\overline{\mathbb{Q}}$, we take $\overline{\mathbb{Q}}$ as an algebraic closure of $F$ and denote the Galois group $\overline{\mathbb{Q}}$ over $F$ by $G_{F}$. Throughout this article, $F$ will be an algebraic number field and $E$ will be a finite Galois extension of $F$ sitting inside $\overline{\mathbb{Q}}$.

In [4], we studied certain subgroups of Brauer groups arising from the twists of matrix algebras. More precisely, let $\operatorname{Hom}_{\text {con }}\left(G_{\mathbb{Q}}, E^{*} / F^{*}\right)$ be the group of all continuous characters from $G_{\mathbb{Q}}$ to $E^{*} / F^{*}$, where the multiplicative group $E^{*} / F^{*}$ is given the discrete topology. Let $\mathrm{Br}(E / F)$ be the subgroup of the Brauer group $\mathrm{Br}(F)$ consisting of all classes of central simple $F$-algebras split by $E$. The theory of twists of matrix algebras defines a natural group homomorphism

$$
\Phi_{E / F}: \operatorname{Hom}_{\mathrm{con}} .\left(G_{\mathbb{Q}}, E^{*} / F^{*}\right) \rightarrow \operatorname{Br}(E / F) .
$$

Our main interest is to find out what the image $H(E / F)$ of $\Phi_{E / F}$ is.

In this article, we shall first give explicit descriptions of the groups $H(E / F)$ for some cyclic extensions $E / F$ in terms of cyclic algebras defined by Gauss sums of Dirichlet characters. Secondly, as an extension of Theorem 5.1 in [4], we shall show that $H(\mathbb{Q}(\sqrt{d}) / \mathbb{Q})=\operatorname{Br}(\mathbb{Q}(\sqrt{d}) / \mathbb{Q})$ for any quadratic field $\mathbb{Q}(\sqrt{d})$. Finally, we shall give examples where $H(E / F)$ are nontrivial proper subgroups of $\operatorname{Br}(E / F)$.

Received 22 November 1993

Supported by NSC Grant \#82-0208-M-007-083, Republic of China.

I would like to express my hearty thanks to the referee for many helpful suggestions.

Copyright Clearance Centre, Inc. Serial-fee code: 0004-9729/94 \$A2.00+0.00. 


\section{Twists of Matrix Algebras}

In this section, we shall recall some basic results on the twists of matrix algebras. For general references, we refer to Section 1, 2 of [2] and Section 2 of [4].

Let $F$ be an algebraic number field contained in $\overline{\mathbb{Q}}$ and take $\bar{F}=\overline{\mathbb{Q}}$ as an algebraic closure of $F$. Let Hom con $_{\text {. }}\left(G_{\mathbb{Q}}, \bar{F}^{*} / F^{*}\right)$ be the group of all continuous characters from $G_{\mathbb{Q}}$ to $\bar{F}^{*} / F^{*}$, where the multiplicative group $\bar{F}^{*} / F^{*}$ is given the discrete topology.

For each $\alpha$ in Hom $_{\text {con }} .\left(G_{\mathbb{Q}}, \bar{F}^{*} / F^{*}\right)$, one can choose a finite Galois extension $E$ of $F$ in $\overline{\mathbb{Q}}$ such that the group $E^{*} / F^{*}$ contains all the values of $\alpha$. Let $\alpha_{F}=\alpha_{\mid G_{F}}$. Then one has the twists $\left(\operatorname{End}_{F} E\right)(\alpha)$ (respectively $\left(\operatorname{End}_{F} E\right)\left(\alpha_{F}\right)$ ) of the matrix algebra $\operatorname{End}_{F} E$ by $\alpha$ (respectively $\alpha_{F}$ ) (see Section 1 of [2]). Moreover, it is known that the central simple $F$-algebras $\left(\operatorname{End}_{F} E\right)(\alpha)$ and $\left(\operatorname{End}_{F} E\right)\left(\alpha_{F}\right)$ are isomorphic (see [2, Proposition (1.2)]).

Let $M(n, \bar{F})$ be the algebra of $n \times n$ matrices over $\bar{F}$. Then, by the SkolemNoether theorem, one can identify the automorphism group $\operatorname{Aut}_{\bar{F}}(M(n, \bar{F}))$ with the projective linear group $P G L(n, \bar{F})$. Let $\Delta_{n}: H^{1}\left(G_{F}, P G L(n, F)\right) \rightarrow H^{2}\left(G_{F}, \bar{F}^{*}\right)=$ $\operatorname{Br}(F)$ be the coboundary operator defined by the following exact sequence of $G_{F^{-}}$ modules:

$$
1 \rightarrow \bar{F}^{*} \rightarrow G L(n, \bar{F}) \rightarrow P G L(n, \bar{F}) \rightarrow 1 .
$$

It is a well-known fact that $\Delta_{n}$ is injective (see $[8$, Theorem 1]). Let $\{\alpha\}$ be the class determined by $\alpha_{F}$ in $H^{1}\left(G_{F}, P G L(n, \bar{F})\right)$. Then the cohomology class of $\left(\operatorname{End}_{F} E\right)(\alpha)$ in $H^{2}\left(G_{F}, \bar{F}^{*}\right)=\operatorname{Br}(F)$ is equal to $\Delta_{n}(\{\alpha\})$, where $n=[E: F]$. Notice that $\{\alpha\}$ is in fact in $H^{1}\left(G_{F}, P G L(n, F)\right)$. Hence the central simple $F$-algebra $\left(\operatorname{End}_{F} E\right)(\alpha)$ is split by $E$.

If $E^{\prime}$ is a finite Galois extension of $F$ containing $E$ in $\overline{\mathbb{Q}}$ with $\left[E^{\prime}: F\right]=m$, then the class $\Delta_{m}(\{\alpha\})$ in $H^{2}\left(\mathrm{Gal}\left(E^{\prime} / F\right), E^{\prime}\right)$ is equal to the inflation of $\Delta_{n}(\{\alpha\})$. This shows that the map $\Phi_{F}:$ Hom $_{\text {con }}\left(G_{\mathbb{Q}}, \bar{F}^{*} / F^{*}\right) \rightarrow H^{2}\left(G_{F}, \bar{F}^{*}\right)=\operatorname{Br}(F)$ defined by $\alpha \longmapsto$ the class of $\left(\operatorname{End}_{F} E\right)(\alpha)$ is well-defined. Moreover, one has the following:

Proposition 2.1. $\Phi_{F}: \mathrm{Hom}_{\text {con }}\left(G_{\mathbb{Q}}, \bar{F}^{*} / F^{*}\right) \rightarrow \operatorname{Br}(F)$ is a group homomorphism. In particular, if $E / F$ is a finite Galois extension, then the subgroup Hom $_{\text {con } .}\left(G_{\mathbb{Q}}, E^{*} / F^{*}\right)$ of the source is mapped by $\Phi_{F}$ into the subgroup $\operatorname{Br}(E / F)$ of the target.

Proof: This follows directly from the definition of $\Phi_{F}$ (see [4, Proposition (2.2)]).

In a recent joint work with $\operatorname{Tan}[5]$, it has been shown that $\Phi_{F}$ is surjective for any algebraic number field $F$. In this article, we are interested in studying the group 
homomorphism $\Phi_{E / F}: \operatorname{Hom}_{\text {con }}\left(G_{\mathbb{Q}}, E^{*} / F^{*}\right) \rightarrow \operatorname{Br}(E / F)$. As we shall see in Section $4, \Phi_{E / F}$ is not surjective in general.

\section{Cyclic extensions $E / F$}

For a finite Galois extension $E / F$ with $\operatorname{Gal}(E / F)=\Gamma$, recall that one can associate each $\alpha$ in Hom $_{\text {con }}\left(G_{\mathbb{Q}}, E^{*} / F^{*}\right)$ to a 2 -cocycle $j_{\alpha}$ on $\Gamma$ with values in $E^{*}$ as follows:

For each $\gamma \in \Gamma$, one defines

$$
\chi_{\gamma}(g)=\frac{\gamma(\dot{\alpha}(g))}{\dot{\alpha}(g)} \text { for all } g \text { in } G_{\mathbb{Q}},
$$

where $\dot{\alpha}(g)$ is a lift of $\alpha(g)$ to $E^{*}$. Then each $\chi_{\gamma}$ is an $E^{*}$-valued Dirichlet character on $G_{\mathbb{Q}}$.

Let $X(E)=\operatorname{Hom}\left(G_{\mathbb{Q}}, E^{*}\right)$ be the $\Gamma$-module of all $E^{*}$-valued Dirichlet characters. For $\chi$ in $X(E)$ of conductor $f$, we define the Gauss sum $\tau(\chi)$ by

$$
\tau(\chi)=\sum_{1 \leqslant a \leqslant f} \chi(a) \zeta_{f}^{a}
$$

where $\zeta_{f}$ is the primitive $f$-th root of unity $e^{2 \pi i / f}$ in $\overline{\mathbb{Q}}^{*}$. For $\chi, \chi^{\prime}$ in $X(E)$, the Jacobi sum $j\left(\chi, \chi^{\prime}\right)$ is defined by $j\left(\chi, \chi^{\prime}\right)=\tau(\chi) \tau\left(\chi^{\prime}\right) / \tau\left(\chi \chi^{\prime}\right)$. It is easy to check that the Jacobi sum takes values in $E^{*}$.

As is well-known [2, Proposition (4.5)], the map $j_{\alpha}: \Gamma \times \Gamma \rightarrow E^{*}$ defined by $j_{\alpha}(\gamma, \delta)=j\left(\chi_{\gamma}^{-1}, \chi_{\delta}^{-\gamma}\right)$ is a 2-cocycle.

In what follows, we assume that $E / F$ is a finite cyclic extension of degree $n$ with $\operatorname{Gal}(E / F)=\Gamma=\langle\sigma\rangle$. Denote by $\chi_{\sigma, \alpha}$ the $E^{*}$-valued Dirichlet character defined by

$$
\chi_{\sigma, \alpha}(g)=\frac{\sigma(\dot{\alpha}(g))}{\dot{\alpha}(g)} \quad \text { for all } g \text { in } G_{\mathbb{Q}} \text {. }
$$

Lemma 3.1. $\left(\operatorname{End}_{F} E\right)(\alpha)$ is isomorphic to the cyclic algebra $(E / F, \sigma, a)$, where $a=\prod_{i=0}^{n-1} \tau\left(\chi_{\sigma, \alpha}^{-\sigma^{i}}\right)$ is an algebraic integer in $F$.

Proof: See [4, Lemma 4.1].

For a cyclic algebra $(E / F, \sigma, a)$, we denote by $[E / F, \sigma, a]$ its class in the Brauer group $\operatorname{Br}(F)$. As an immediate consequence, one has the following:

COROLLaRY 3.2. Let $E / F$ be a finite cyclic extension of degree $n$ with $\operatorname{Gal}(E / F)=\Gamma=\langle\sigma\rangle$. Then

$$
H(E / F)=\left\{\left[E / F, \sigma, \prod_{i=0}^{n-1} \tau\left(\chi_{\sigma, \alpha}^{-\sigma^{i}}\right)\right] \mid \alpha \in \text { Hom }_{\text {con } .}\left(G_{Q}, E^{*} / F^{*}\right)\right\}
$$


For each $n \geqslant 1$, let $\mu_{n}=\left\langle\zeta_{n}\right\rangle$ be the group of $n$-th roots of unity, where $\zeta_{n}$ is a primitive $n$-th root of unity. For an algebraic number field $F$, let $\mu(F)$ be the group of roots of unity in $F$. For the rest of this section, we shall consider the following special types of cyclic extensions.

Assume that $\mu_{n} \subset F$. Let $a \in F^{*}$ be such that $\left[F\left({ }^{n} \sqrt{a}\right): F\right]=n$. Then $F(\sqrt[n]{a})$ is a cyclic extension of $F$ of degree $n$ and $\sigma: \sqrt[n]{a} \longmapsto \sqrt{a} \sqrt{a} \zeta_{n}$ is the generating automorphism of the Galois group $\operatorname{Gal}\left(F\left({ }^{n} \sqrt{a}\right) / F\right)$.

Consider the map

$$
\psi: \operatorname{Hom}_{\mathrm{con}} \cdot\left(G_{\mathbb{Q}}, F\left({ }^{n} \sqrt{a}\right)^{*} / F^{*}\right) \rightarrow X\left(F\left(^{n} \sqrt{a}\right)\right)
$$

defined by

$$
\psi(\alpha)=\chi_{\sigma, \alpha}
$$

Lemma 3.3. If $\mu\left(F\left({ }^{n} \sqrt{a}\right)\right)=\mu(F)=\left\langle\zeta_{n}\right\rangle$, then $\psi$ is surjective.

PROOF: For any $F\left({ }^{n} \sqrt{a}\right)^{*}$-valued Dirichlet character $\chi$, let $\operatorname{ker} \chi=\mathrm{Gal}(\overline{\mathbb{Q}} / H)$ such that $\chi: \operatorname{Gal}(H / \mathbb{Q}) \stackrel{\sim}{\longrightarrow}\left\langle\zeta_{n}^{h}\right\rangle$ for some integer $h, 0 \leqslant h \leqslant n-1$.

Let $\mathrm{Gal}(H / \mathbb{Q})=\langle\tau\rangle$ and let $\pi: G_{\mathbb{Q}} \rightarrow \mathrm{Gal}(H / \mathbb{Q})$ be the canonical surjection. Define the map $\beta: \operatorname{Gal}(H / \mathbb{Q}) \rightarrow F(\sqrt[n]{a})^{*} / F^{*}$ by sending $\tau$ to $(\sqrt{a})^{h} \bmod F^{*}$. Then $\alpha=\beta \circ \pi$ is a continuous character from $G_{\mathbb{Q}}$ to the discrete group $F\left({ }^{n} \sqrt{a}\right)^{*} / F^{*}$. By definition, it is easy to see that $\chi_{\sigma, \alpha}=\chi$.

REMARK. Under the assumption of Lemma 3.3, $X\left(F\left({ }^{n} \sqrt{a}\right)\right)=X(F)$.

THEOREM 3.4. Suppose that $\mu\left(F\left({ }^{n} \sqrt{a}\right)\right)=\mu(F)=\left\langle\zeta_{n}\right\rangle$ and $\left[F\left({ }^{n} \sqrt{a}\right): F\right]=n$. Then $H(F(\sqrt[n]{a}) / F)=\left\{\left[F(\sqrt[n]{a}) / F, \sigma, \tau(\chi)^{n}\right] \mid \chi \in X(F)\right\}$.

Proof: By Corollary 3.2 , each class in $H\left(F\left({ }^{n} \sqrt{a}\right) / F\right)$ is represented by a cyclic algebra of the form

$$
\left(F(\sqrt[n]{a}) / F, \sigma, \prod_{i=0}^{n-1} \tau\left(\chi_{\sigma, \alpha}^{-\sigma^{i}}\right)\right) \text { for some } \alpha \in \text { Hom }_{\text {con }} .\left(G_{\mathbb{Q}}, F(\sqrt[n]{a})^{*} / F^{*}\right) .
$$

By the assumption that $\mu\left(F\left({ }^{n} \sqrt{a}\right)\right)=\mu(F)$, one has that $\chi_{\sigma, \alpha}^{-\sigma^{i}}=\chi_{\sigma, \alpha}^{-1}$ for each $i=$ $0,1, \cdots, n-1$. The assertion follows immediately from Lemma 3.3.

For $a, b$ in $F^{*}$, let $(a, b) / F$ be the quaternion algebra generated by $a, b$ (see [6, p.78]). Denote by $\left[\left(\frac{a, b}{F}\right)\right]$ its class in $\operatorname{Br}(F)$.

Corollary 3.5. Let $F$ be a totally real number field and let $a \in F^{*}-F^{*^{2}}$ be such that $\mu(F)=\mu(F(\sqrt{a}))=\{ \pm 1\}$. Then

$$
H(F(\sqrt{a}) / F)=\left\{\left[\left(\frac{a, n}{F}\right)\right] \mid n \in \mathbb{Z}\right\}
$$


Proof: By Theorem 3.4, $H(F(\sqrt{a}) / F)=\left\{\left[F(\sqrt{a}) / F, \sigma, \tau(\chi)^{2}\right] \mid \chi\right.$ runs over all quadratic Dirichlet characters $\}$.

On the other hand, it is well known that $\tau(\chi)^{2}$ runs over the set of integers \{ \pm odd primes, $\pm 8,-4\}$ (see [9, p.28, Exercise 3.5] and [1, p.349, Theorem 7]). Our assertion follows immediately from elementary facts on cyclic algebras (see [7, p.260, Theorem (30.4)] and [6, p.79, Lemma 2]).

COROLlary 3.6. Let $\mathbb{Q}(\sqrt{d})$ be a quadratic field such that $d \neq-1,-3$. Then

$$
H(\mathbb{Q}(\sqrt{d}) / \mathbb{Q})=\operatorname{Br}(\mathbb{Q}(\sqrt{d}) / \mathbb{Q})
$$

Proof: Let Gal $(\mathbb{Q}(\sqrt{d}) / \mathbb{Q})=\{\sigma$, id. $\}$. By Br $(\mathbb{Q}(\sqrt{d}) / \mathbb{Q}) \simeq \mathbb{Q}^{*} / N\left(\mathbb{Q}(\sqrt{d})^{*}\right)$ where $N$ is the norm from $\mathbb{Q}(\sqrt{d})$ to $\mathbb{Q}$, each class in $\operatorname{Br}(\mathbb{Q}(\sqrt{d}) / \mathbb{Q})$ is represented by a cyclic algebra of the form

$$
(\mathbb{Q}(\sqrt{d}) / \mathbb{Q}, \sigma, n), \quad \text { where } n \in \mathbb{Z}
$$

Hence the assertion follows from Corollary 3.5 and an elementary fact on cyclic algebras (see [6, p.79, Lemma 2]).

Although $d=-1,-3$ were excluded in Corollary 3.6, one may prove the following theorem directly without using $(3.3) \sim(3.5)$.

THEOREM 3.7. For any quadratic field $Q(\sqrt{d}), H(\mathbb{Q}(\sqrt{d}) / \mathbb{Q})=$ $\operatorname{Br}(\mathbb{Q}(\sqrt{d}) / \mathbb{Q})$.

Proof: Let Gal $(\mathbb{Q}(\sqrt{d}) / \mathbb{Q})=\{\sigma$, id. $\}$, where $\sigma(\sqrt{d})=-\sqrt{d}$. Recall that each class in $\operatorname{Br}(\mathbb{Q}(\sqrt{d}) / Q)$ is represented by a cyclic algebra of the form $(\mathbb{Q}(\sqrt{d}) / \mathbb{Q}, \sigma, n)$ for some $n \in \mathbb{Z}$.

For any quadratic field $\mathbb{Q}(\sqrt{D})$ of discriminant $D$, one considers the continuous character $\alpha$ in Hom $_{\text {con }}\left(G_{\mathbb{Q}}, \mathbb{Q}(\sqrt{d})^{*} / \mathbb{Q}^{*}\right)$ defined by the natural map $G_{\mathbb{Q}} \rightarrow$ $\operatorname{Gal}(\mathbb{Q}(\sqrt{D}) / \mathbb{Q})$ and the natural embedding $\tau \longmapsto \sqrt{d} \bmod \mathbb{Q}^{*}$ of $\operatorname{Gal}(\mathbb{Q}(\sqrt{D}) / \mathbb{Q})$ into $\mathbb{Q}(\sqrt{d})^{*} / \mathbb{Q}^{*}$, where $\tau \in \mathrm{Gal}(\mathbb{Q}(\sqrt{D}) / \mathbb{Q})$ is such that $\tau(\sqrt{D})=-\sqrt{D}$. Let $\chi_{\sigma, \alpha}$ be the Dirichlet character defined by

$$
\chi_{\sigma, \alpha}(g)=\frac{\sigma(\dot{\alpha}(g))}{\dot{\alpha}(g)} \quad \text { for all } g \in G_{\mathbf{Q}}
$$


where $\dot{\alpha}(g)$ is a lift of $\alpha(g)$. Then it is easy to see that $\chi_{\sigma, \alpha}$ is the quadratic character associated to the quadratic field $\mathbb{Q}(\sqrt{D})$.

On the other hand, by Lemma 3.1, $\left(\operatorname{End}_{\mathbb{Q}} \mathbb{Q}(\sqrt{d})\right)(\alpha)$ is isomorphic to the cyclic algebra $\left(\mathbb{Q}(\sqrt{d}) / \mathbb{Q}, \sigma, \tau\left(\chi_{\sigma, \alpha}\right)^{2}\right)$. As we have mentioned above, it is well-known that $\tau\left(\chi_{\sigma, \alpha}\right)^{2}=D$ and $D$ runs over the set of integers $\{ \pm$ odd primes, $\pm 8,-4\}$. Our assertion follows from elementary facts on cyclic algebras.

\section{EXAMPLES}

Let $n \geqslant 1$ be an integer such that $\varphi(n)$ (the Euler $\varphi$-function) has an odd prime factor $p$. Let $E=\mathbb{Q}\left(\zeta_{n}\right)$ and $F=\mathbb{Q}$. For a quadratic subfield $\mathbb{Q}(\sqrt{d})$ in $\mathbb{Q}\left(\zeta_{n}\right)$, it is easy to see that $H(\mathbb{Q}(\sqrt{d}) / \mathbb{Q}) \subseteq H\left(\mathbb{Q}\left(\zeta_{n}\right) / \mathbb{Q}\right)$. On the other hand, it is known that $H\left(\mathbb{Q}\left(\zeta_{n}\right) / \mathbb{Q}\right) \subseteq \operatorname{Br}\left(\mathbb{Q}\left(\zeta_{n}\right) / \mathbb{Q}\right) \cap S(\mathbb{Q})$,

where $S(\mathbb{Q})=\mathrm{Br}_{2}(\mathbb{Q})$ is the Schur subgroup of $\mathrm{Br}(\mathbb{Q})$ (see [3, Theorem 3.1] and [10, Theorem 7.2]). Moreover, the group $\operatorname{Br}\left(\mathbb{Q}\left(\zeta_{n}\right) / \mathbb{Q}\right)$ clearly has an element of order $p$.

One concludes that $B\left(\mathbb{Q}\left(\zeta_{n}\right) / \mathbb{Q}\right)$ is a nontrivial proper subgroup of $\operatorname{Br}\left(\mathbb{Q}\left(\zeta_{n}\right) / \mathbb{Q}\right)$.

\section{REFERENCES}

[1] Z.I. Borevich and I.R. Shafarevich, Number theory (Academic Press, New York, 1966).

[2] W. Chi, 'Twists of central simple algebras and endomorphism algebras of some Abelian varieties', Math. Ann. 276 (1987), 615-632.

[3] W. Chi, 'Hasse invariants of some endomorphism algebras of Abelian varieties', Chinese J. Math. 18 (1990), 87-94.

[4] W. Chi, 'Twists of matrix algebras and some subgroups of Brauer groups', Bull. Austral. Math. Soc. 45 (1992), 377-383.

[5] W. Chi and K.S. Tan, 'Twists of matrix algebras and Brauer groups', preprint.

[6] P.K. Draxl, Skew fields (Cambridge University Press, Cambridge, 1983).

[7] I. Reiner, Maximal orders (Academic Press, New York, 1975).

[8] P. Roquette, 'On the Galois cohomology of the projective linear group and its applications to the construction of generic splitting fields of algebras', Math. Ann. 150 (1963), 411-439.

[9] L.C. Washington, Introduction to cyclotomic fields (Graduate Texts in Mathematics 83, Springer-Verlag, Berlin, Heidelberg, New York, 1982).

[10] T. Yamada, The Schur subgroup of the Brauer group, Lecture Notes in Math. 397 (Springer-Verlag, Berlin, Heidelberg, New York, 1974).

Department of Mathematics

National Tsing Hua University

Hsinchu, Taiwan 30043

Republic of China 